\title{
Uncertainty Is Associated With Biased Expectancies and Heightened Responses to Aversion
}

\author{
Daniel W. Grupe and Jack B. Nitschke \\ University of Wisconsin-Madison
}

\begin{abstract}
Uncertainty is an omnipresent force in peoples' lives that has been shown to amplify the negative impact of aversive events. This amplified aversiveness, together with the negative attitudes that individuals can have toward uncertainty, suggests that a cue indicating uncertainty about future events might be associated with biased expectancies of negative outcomes or biased contingency estimates, similar to biases that have been observed for traditional fear-relevant cues, such as snakes or spiders. Participants in this study saw three different cues: one that indicated with certainty that an aversive picture would follow, one that indicated with certainty that a neutral picture would follow, and one that indicated uncertainty about whether an aversive or neutral picture would follow. Online self-report data revealed negatively biased expectancies of aversion after uncertain cues. The degree of this online expectancy bias predicted participants' estimates, at the conclusion of the experiment, of the relationship between uncertain cues and aversive pictures. Aversive pictures after the uncertain cue (relative to those after the certain cue) were accompanied by increased skin conductance responses and self-reported negative mood. These findings that uncertainty is accompanied by biased expectancies of aversion and heightened responses to aversion warrant extensions of this research in anxiety disorders, given evidence for intolerance of uncertainty and anticipatory dysfunction in the pathology of such disorders.
\end{abstract}

Keywords: uncertainty, expectancy bias, skin conductance, aversion, emotion

Humans are bombarded with uncertainty throughout their everyday lives, and resolving this uncertainty is a primary motive in peoples' lives (Kagan, 1972). The resolution of uncertainty occurs through the identification of relationships between environmental cues and subsequent outcomes (Alloy \& Tabachnik, 1984). The identification of such contingencies can provide individuals with more certainty about the future and promote the achievement of desired outcomes. Awareness of relationships between specific cues and subsequent events can also be generalized or applied to similar circumstances, resulting in further reduction of uncertainty about future events. The identification of environmental contingencies is a critical adaptive ability that has clear survival benefits, because it allows organisms to explain past events, control current happenings, and prepare for the future (Alloy \& Tabachnik, 1984). Despite the importance of this skill, humans are notoriously flawed

Daniel W. Grupe and Jack B. Nitschke, Departments of Psychology and Psychiatry, University of Wisconsin-Madison.

Thanks to Danielle Green, Craig Marquardt, Melissa Schmidt, Kaitlin McLean, Larry Greischar, and Adam Koppenhaver for help with data collection and processing; to John Govin, Andy Mulder, and Nicholas Balderston for helping to design and build the response dial; and to Lyn Abramson, John Curtin, Brad Postle, and Desmond Oathes for comments and advice on this article. This research was supported in part by grants from the National Institute of Mental Health (R01 MH07487 and Training Grant T32-MH018931), by a National Science Foundation Graduate Research Fellowship, and by a Menzies research award through the UW Department of Psychology.

Correspondence concerning this article should be addressed to Daniel W. Grupe, Waisman Laboratory for Brain Imaging and Behavior, 1500 Highland Avenue, Madison, WI 53705. E-mail: grupe@wisc.edu at objective contingency awareness and frequently misjudge relationships between cues and resulting outcomes. Factors such as memory load, the salience of events, the presence of motivational factors, and the availability and complexity of situational information all have an impact on one's ability to objectively assess such contingencies (for review, see Arkes \& Harkness, 1983).

Previous expectancies regarding the relationship between cues and outcomes can interfere with the objective assessment of contingencies, particularly in the absence of explicitly presented or readily accessible information about such contingencies (Alloy \& Tabachnik, 1984). In one representative paradigm designed to investigate contingency awareness, participants are presented with cues that are either fear-relevant (e.g., pictures of spiders) or fear-irrelevant (e.g., pictures of mushrooms), which are then followed by either aversive or neutral outcomes (e.g., electric shock vs. neutral auditory tone). At the onset of such experiments, individuals already have inflated expectancies (i.e., $>50 \%$ ) of aversive outcomes after fear-relevant cues, an effect deemed "a priori expectancy bias" (Davey, 1992, 2006). These biased expectancies frequently persist after multiple presentations of fearrelevant and fear-irrelevant cues that are equally likely to precede aversive and neutral outcomes, as evidenced by online expectancy ratings (Amin \& Lovibond, 1997; de Jong, Merckelbach, \& Arntz, 1995; de Jong, Merckelbach, Bögels, \& Kindt, 1998). Heightened expectancies are particularly pronounced in populations selected for high fear of the fear-relevant cue (de Jong et al., 1995; de Jong et al., 1998; Engelhard, de Jong, van den Hout, \& van Overveld, 2009; Pauli, Wiedemann, Dengler, \& Kühlkamp, 2001; Wiedemann, Pauli, \& Dengler, 2001), although expectancy biases have also been demonstrated in unselected, nonphobic participants 
(Amin \& Lovibond, 1997; Davey, 1992; Davey \& Dixon, 1996; de Jong et al., 1998; Kennedy, Rapee, \& Mazurski, 1997; McNally \& Heatherton, 1993; Mühlberger, Wiedemann, Herrman, \& Pauli, 2006).

Biased expectancies of aversive outcomes take on greater importance in light of the dramatic influence that expectancies can have on the perception of and behavioral responses to a diverse array of events and their associated underlying neural processes. The most salient examples may be placebo effects in drug studies or the treatment of pain (Atlas, Bolger, Lindquist, \& Wager, 2010; Volkow et al., 2006; Wager \& Nitschke, 2005; Wager et al., 2004; Zubieta et al., 2005), but altering expectancies can similarly affect primary sensory responses to olfactory and gustatory stimuli (de Araujo, Rolls, Velazco, Margot, \& Cayeux, 2005; Nitschke et al., 2006a; Sarinopoulos, Dixon, Short, Davidson, \& Nitschke 2006), the perceived pleasantness of art and wine (Kirk, Skov, Hulme, Christensen, \& Zeki, 2009; Plassman, O’Doherty, Shiv, \& Rangel, 2008), and social decision-making (Sanfey, 2009), to name a few examples.

Expectancy biases show strong resistance to disconfirmation, particularly in high-fear individuals (Davey, 2006), and can contribute to "illusory correlations" between unrelated events even after extensive exposure to different cues and outcomes (Chapman \& Chapman, 1967, 1969). In paradigms such as that described above, numerous studies have identified a "covariation bias," that is, an overestimate (at the conclusion of the experiment) of the covariance of fear-relevant cues and aversive outcomes (Amin \& Lovibond, 1997; de Jong et al., 1995; Hermann, Ofer, \& Flow, 2004; Pauli, Wiedemann, \& Montoya, 1998; Tomarken, Mineka, \& Cook, 1989). This bias is specific to the combination of fearrelevant cues and aversive outcomes; in each of the studies cited above, subjects provided accurate covariation estimates for fearrelevant cues and nonaversive outcomes, as well as for fearirrelevant cues and aversive outcomes. It is unclear to what extent this covariation bias is attributable to an a priori expectancy bias, as opposed to online biases in the way corroborating or conflicting evidence is processed (Amin \& Lovibond; Davey \& Dixon, 1996; McNally \& Heatherton, 1993; Tomarken et al.). However, high correlations between a priori expectancies, online expectancies, and postexperiment covariation estimates (Amin \& Lovibond, 1997; de Jong et al., 1995, 1998) suggest that these biases likely reflect similar underlying processes.

For some individuals, uncertainty about the future can be an intolerable state that interferes with their ability to adequately prepare for potentially negative events and that causes such events to seem even more stressful or aversive (Bar-Anan, Wilson, \& Gilbert, 2009; Hirsh \& Inzlicht, 2008; Kimmel, 1967; Nader \& Balleine, 2007). This is especially true for anxiety disorders, particularly generalized anxiety disorder, for which intolerance of uncertainty has been put forth as a core construct (Dugas, Gagnon, Ladouceur, \& Freeston, 1998). These negative attitudes and enhanced responses may be seen in situations involving both risk (i.e., multiple possible outcomes with known probabilities) as well as ambiguity (i.e., unknown probabilities of event occurrences). Accordingly, the present study allows for the possibility of both risk and ambiguity rather than attempting to distinguish between them (Bach, Seymour, \& Dolan, 2009; Hsu, Bhatt, Adolphs, Tranel, \& Camerer, 2005).
A cue indicating uncertainty about the probability of an upcoming aversive stimulus may serve a role akin to the fear-relevant cues used in the aforementioned illusory correlation studies. We recently conducted a functional magnetic resonance imaging (fMRI) study (Sarinopoulos et al., 2010) in which subjects viewed aversive pictures either in the context of certainty (preceded by a cue indicating an aversive picture would definitely appear) or uncertainty (preceded by a cue indicating that an aversive picture might appear). ${ }^{1}$ After the fMRI scan, subjects were asked to estimate the percentage of uncertain cues that were followed by aversive pictures. Although uncertain cues were equally likely to be followed by aversive or neutral pictures, subjects reported that these cues were significantly more likely to be followed by aversive pictures. ${ }^{2}$ In addition, the degree of participants' postexperimental bias was correlated with activity in two key brain regions: the anterior cingulate cortex (ACC), which frequently shows activation during anticipation of aversion and regulation of negative emotion (Etkin, Egner, Peraza, Kandel, \& Hirsch, 2006; Ploghaus, Becerra, Borras, \& Borsook, 2003; Sarinopoulos et al., 2006), and the insula, which is posited to play a key role in interoception and the representation of emotional states (see Craig, 2009, for review). Specifically, participants' postexperiment estimates were positively associated with ACC activation to uncertain cues and negatively related to insula responses to subsequent aversive pictures, possibly reflecting enhanced ACC regulatory functions and compromised interoceptive functions of the insula. Interestingly, these same brain regions have been implicated in the pathology of anxiety disorders (Etkin \& Wager, 2007; Etkin, Prater, Hoeft, Menon, \& Schatzberg, 2010; Nitschke et al., 2009; Paulus \& Stein, 2006), which are characterized in part by the excessive anticipation of threat (Barlow, 2002; Nitschke et al., 2009) as well as worry about the future and intolerance of uncertainty (Borkovec \& Inz, 1990; Dugas et al., 1998).

The findings of Sarinopoulos et al. (2010) raised the possibility that individuals with biased expectancies of aversive events after the uncertain cue may show altered activation (relative to individuals with unbiased expectancies) in regions that are involved in the

\footnotetext{
${ }^{1}$ To frame this study (as well as the current study, which uses the same paradigm) in the context of research on behavioral economics and neuroeconomics, this uncertain condition most resembled ambiguity at the outset of the experiment because participants had incomplete information regarding the probabilities of outcomes associated with the uncertain cue. Over the duration of the experiment, subjects acquired greater information about these probabilities, and the uncertain condition likely came to more closely resemble a risky situation.

${ }^{2}$ Note that there are a number of important distinctions between this paradigm and a typical illusory correlation paradigm used to investigate expectancy/covariation biases. For example, in the current study, there is only one cue with multiple outcomes, and the postexperiment questionnaire only queried participants about this cue-outcome relationship. In addition, participants were not instructed to attend to the relative percentages of aversive and neutral pictures after the uncertain cue, as is standard procedure in illusory correlation paradigms. There is, however, a striking similarity between this effect and the traditionally defined "covariation bias" (Tomarken et al., 1989) in that a fear/anxiety-relevant cue with multiple outcomes of equal probability was more likely to be associated with aversive than neutral outcomes. The literature on biased expectancies and estimates of covariation is thus highly relevant to the findings of Sarinopoulos et al. (2010), as well as to the current investigation.
} 
anticipation of and response to aversive events (Dunsmoor, Bandettini, \& Knight, 2008; Nitschke et al., 2006b; Ploghaus et al., 1999). However, because subjects were only asked at the conclusion of the experiment to estimate the relationship between uncertain cues and aversive pictures, it is unclear whether this bias was primarily attributable to an a priori expectancy bias, biased online expectancies after the uncertain cue, or continued offline analysis or distorted memories of cue-outcome relationships. The current study was designed to resolve this issue by identifying whether uncertainty results in biased expectancies of aversion and whether such an expectancy bias would be reflected in participants' subsequent responses to aversive events.

To investigate the genesis and development of biased estimates of aversion under conditions of uncertainty, we continuously monitored participants' self-reported expectancies of aversive pictures (de Jong et al., 1995) after cues indicating certainty or uncertainty about whether an aversive picture would appear. Immediately after the picture paradigm, participants were asked to estimate the relationship between the uncertain cue and aversive pictures. On the basis of the conceptualization of uncertainty as a fear-relevant/ aversive state and on the findings reviewed above that demonstrate altered expectancies and covariation estimates related to fearrelevant cues (Davey, 1992; de Jong et al., 1995; Tomarken et al., 1989), we expected to see uncertainty-related biases in a priori and online expectancy ratings as well as postexperiment estimates, such that uncertain cues would be overassociated with aversive pictures. We compared skin conductance responses (SCRs) and online mood ratings in response to aversive pictures that followed uncertain versus certain cues. In line with reports suggesting that uncertainty can intensify the negative impact of aversive stimuli (Bar-Anan, Wilson, \& Gilbert, 2009; Hirsh \& Inzlicht, 2008; Kimmel, 1967; Nader \& Balleine, 2007), we hypothesized that participants would demonstrate stronger emotional responses to aversive pictures after uncertain cues than to aversive pictures after certain cues. Finally, by enrolling a separate group of participants who did not rate their online expectancies of aversive pictures, we assessed whether the act of reporting expectancies measurably affected processes intrinsic to the task.

\section{Method}

\section{Participants}

Participants for this study were 84 individuals (40 female; mean age $\pm S D=24.5 \pm 5.3$ years) who responded to flyers posted on and around the University of Wisconsin-Madison campus. This sample consisted primarily of undergraduate and graduate students (54 White, 18 Asian, 4 Black, 1 Hispanic, 1 American Indian, and 6 more than one race). Participants reported no current or past history of psychiatric problems for themselves or their first-degree relatives and were not currently taking medication used to treat a psychiatric condition. Individuals were compensated $\$ 40$ for their participation at the completion of the study. Of these 84 participants, 5 individuals did not demonstrate an understanding of the task instructions or complete the task as instructed and 1 withdrew early, resulting in a final sample of 78 participants (38 female; mean age $\pm S D=24.6 \pm 5.1$ years). Participants were randomly assigned to one of two groups (described below) at the time of enrollment: an "Expectancy Ratings" (ER) group $(N=39,18$ female) and a "No Expectancy Ratings" (NER) group ( $N=39,20$ female). All participants were right-handed, as assessed by the Edinburgh Handedness Inventory (Oldfield, 1971), or preferred to use their right hand to manipulate the dial used during the experiment. All participants provided informed consent in accord with study approval by the UW Health Sciences Institutional Review Board.

\section{Experimental Design and Stimuli}

Participants took part in a cued picture task similar to that used by Sarinopoulos et al. (2010), in which one of three anticipatory cues preceded either a neutral or aversive picture from the International Affective Picture System (IAPS; Lang, Bradley, \& Cuthbert, 2008). The aversive pictures selected for this study were the most aversive (e.g., mutilated bodies, accident scenes) in the set based on published norms (Lang et al., 2008). Photos in which the content of the slide was difficult to discern at the 1-s duration used in the experiment (as determined by the experimenters) were excluded from use. On the 0 to 9 scale used by Lang et al. (2008), aversive pictures had a mean arousal rating of $6.05(S D=0.80)$ and mean valence rating of $2.32(S D=0.71)$. Neutral pictures had a mean arousal rating of $3.26(S D=0.76)$ and mean valence rating of $5.45(S D=0.81)$. The design and timing of the paradigm, as well as the selection of stimuli, were patterned after the fMRI experiment of Sarinopoulos et al. (2010) to facilitate comparison with the results of that study.

Each trial (see Figure 1) consisted of a 2-s cue that warned participants about the upcoming picture. There were three different warning cues, each of which was white on a black background: an " $\mathrm{X}$ " cue, which always preceded aversive pictures, an "O" cue, which always preceded neutral pictures, and a "?" cue, which could precede either aversive or neutral pictures. Participants were provided with information about the relationships between cues and pictures but were not told that the "?" cue preceded neutral and aversive pictures at exactly a 50/50 ratio. For participants in the ER group only, this cue was immediately followed by a 4-s expectancy rating scale. The prompt "Expect Aversive Picture?" appeared above a visual analogue scale from 0 to 100 . On this scale, 0 was labeled "expect neutral," 50 was labeled "uncertain," and 100 was labeled "expect aversive." Participants were instructed to rate their expectancy that an aversive picture would appear by turning a dial with their right hand to move a cursor along this scale. Participants were told to move the cursor closer to one end or the other depending on how certain they were of their decision. Individuals in the NER group did not see this expectancy ratings scale and received no additional task instructions after presentation of the cue.

The expectancy rating scale (ER group) or the cue (NER group) was followed by a variable-length interstimulus interval (ISI) of 4 to $10 \mathrm{~s}$ and then a $1-\mathrm{s}$ presentation of a neutral or aversive picture. This picture was followed by a 5- to 7-s ISI and then a 4-s mood or picture rating scale. When this scale appeared, participants used the dial to rate either their mood or the picture valence on a visual analogue scale (only 1 rating per trial). Both scales were labeled as follows: $-4=$ "unpleasant/negative," $0=$ "neutral," $+4=$ "pleasant/happy." For all rating scales (expectancy, mood, and picture), the position of the cursor on the scale was recorded every $100 \mathrm{~ms}$ during each rating period. Before analysis of self-report 


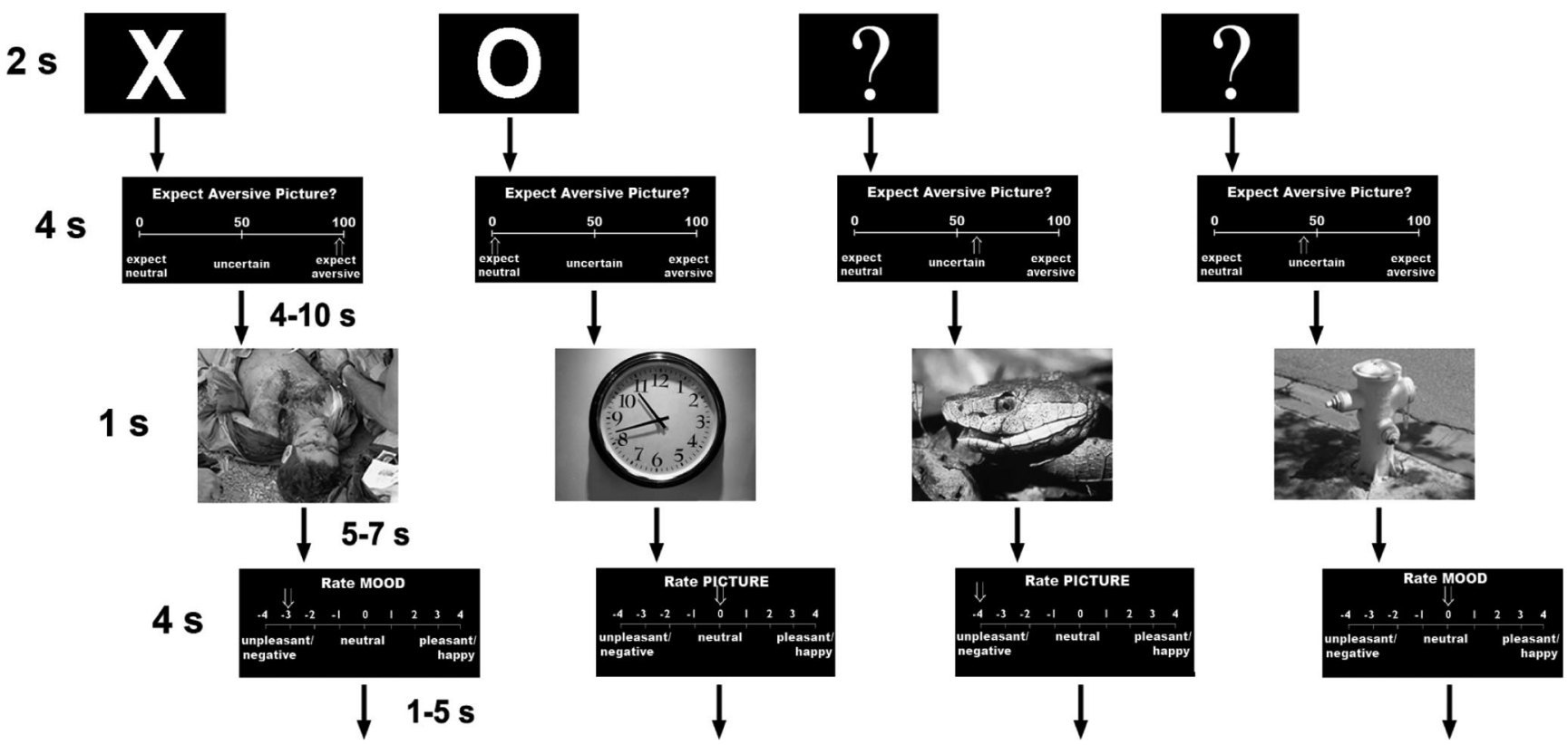

Figure 1. Schematic representation of the trial structure for each of the four possible trial types. Each column represents a separate trial. Because of copyright issues, pictures presented here are from the public domain and approximate the type of pictures presented during the experiment.

data, $x$-coordinates for the cursor position over the last $500 \mathrm{~ms}$ of each rating were linearly transformed into the appropriate numerical measure ( -4 to +4 for picture and mood ratings; $0 \%$ to $100 \%$ for the expectancy ratings). A 1 - to 5-s intertrial interval (ITI) followed the offset of the rating scale. Each ISI and ITI consisted of a black background with a centrally presented white crosshair, on which participants were instructed to fixate.

There were four experimental runs, each of which consisted of 8 certain aversive trials, 8 certain neutral trials, and 8 uncertain trials. Over the course of the experiment, participants viewed 32 aversive and 32 neutral pictures after the certain cues ("X" and "O," respectively), as well as 16 aversive and 16 neutral pictures after the uncertain cue ("?"). Pictures occurring after the certain and uncertain cues were equated on dimensions of arousal and valence using normative ratings (Lang et al., 2008). In addition, each run included four to five trials in which one of the three cues was presented with no subsequent picture, as well as three to four pictures presented at the end of the run with no preceding warning cue (these trials are included in our current fMRI research using this paradigm and were not analyzed for the present study). Each run lasted approximately 12 min, and participants were given a short break between runs. The uncertain cues were followed by one of two pseudorandom trial orders of aversive and neutral pictures to avoid trial order effects that may have influenced participants' estimates of cue-picture relationships (de Jong \& Merckelbach, 1990; Wiens, Katkin \& Öhman, 2003). Within each of these two trial orders, individual IAPS stimuli were shuffled between participants to generate three different cue-picture orders, such that pictures after the certain cue for two-thirds of the participants followed the uncertain cue for the other one-third. This was done to ensure that effects attributed to the certainty manipu- lation were not simply caused by interstimulus differences in valence or arousal. In all, this resulted in six different experiment versions, which were counterbalanced across participants in both the ER and NER groups.

\section{Procedure and Apparatus}

Data collection took place in the electrophysiological suite in the Waisman Laboratory for Brain Imaging and Behavior on the UW-Madison campus. After informed consent, two shielded Ag/ $\mathrm{AgCl}$ electrodes $(4 \mathrm{~mm})$ were filled with $\mathrm{NaCl}$ paste and attached to the distal phalanges of participants' left index and middle fingers. Skin conductance was monitored during the experiment using the BIOPAC MP150 System (BIOPAC Systems Inc., Goleta, CA) at a sampling rate of $1000 \mathrm{~Hz}$, with a $10 \mathrm{~Hz}$ low-pass filter. Participants entered a sound-attenuated, radio-frequency shielded booth and were placed approximately 24" from a 20" LCD monitor on which stimuli were presented using E-Prime software (Psychology Software Tools, Pittsburgh, PA). Participants completed a brief practice version of the experiment, immediately followed by the four experimental runs. At the completion of the final experimental run, participants were removed from the experimental booth and immediately administered a poststudy questionnaire by the experimenter, which included the question "What percentage of the question mark cues were followed by aversive events?" Responses to this question are referred to below as "postexperiment estimates". After the administration of this questionnaire, participants completed the Intolerance of Uncertainty Scale (IUS; Buhr \& Dugas, 2002), which assesses how stressful, upsetting, negative, or incapacitating the state of uncertainty is for individuals. 


\section{Data Analysis}

Self-report data. Three bias measures were assessed in this experiment: a priori, online, and postexperiment biases. In each case, the relevant self-report measure was tested against $50 \%$, with a score greater than $50 \%$ reflecting one of the hypothesized biases related to the uncertain cue/aversive picture pairing. Expectancy ratings for the initial "?" cue were tested against 50\% using a one-sample $t$ test in the ER group to test for an a priori expectancy bias (Davey, 1992). For the online expectancy bias, total expectancy ratings (averaged across all runs but not including the initial trial) for the "?" cue were tested against 50\% using a one-sample $t$ test in the ER group. Expectancy ratings from runs 1 to 4 were multiplied by linear contrast coefficients $(-3,-1,+1,+3)$ to test for a linear trend in expectancy ratings after the "?" cue over the four runs. For the postexperiment bias, a one-sample $t$ test collapsed across groups assessed whether postexperiment estimates of the relationship between the uncertain cue and aversive pictures differed from $50 \%$.

To test the hypothesis that uncertainty would be associated with heightened self-report of negative affect after aversive pictures, a paired two-sample $t$ test compared mood ratings for aversive pictures preceded by certain ("X") versus uncertain ("?") cues. The final study question-whether the act of reporting expectancies measurably affected processes intrinsic to the task-was tested by an omnibus Group (ER, NER) $\times$ Certainty (Certain, Uncertain) $\times$ Picture Valence (Aversive, Neutral) repeated-measures analysis of variance (ANOVA) for the mood ratings following aversive pictures preceded by "X" or "?" and after neutral pictures preceded by "O" or "?." Analogous analyses were conducted for ratings of picture valence.

Skin conductance data. With use of custom functions (courtesy of Larry Greischar) written for MATLAB (The MathWorks, Natick, MA), each run was linearly detrended to account for the commonly observed decrease in skin conductance activity across the experiment. The four runs were concatenated, and data were mean-filtered by averaging the signal at each time point with the 150 samples around that time point to reduce the impact of brief duration signal spikes. Discrete SCRs were identified by examining the skin conductance signal, which was time-locked to the onset of each picture. A SCR was defined as a continuous positive deflection of at least $0.03 \mu \mathrm{S}$ that started between 0.5 and $4 \mathrm{~s}$ after picture onset, and that peaked before $8 \mathrm{~s}$ after picture onset. SCRs were square root-transformed to eliminate positive skewness and normalize the distribution of responses.

A measure of SCR magnitude was calculated for each condition by averaging SCRs for all events, including zeros for responses that failed to meet the SCR threshold of $0.03 \mu \mathrm{S}$ noted above. The use of the magnitude measure, which averages SCRs over all trials (as opposed to a amplitude measure, which restricts analysis to trials on which a suprathreshold response is recorded), can confound response frequency with response amplitude (Dawson, Schell, \& Filion, 2007; Urry, van Reekum, Johnstone, \& Davidson, 2009). To exclude infrequent responders from our analysis, only participants with discernible suprathreshold SCRs to at least $25 \%$ of pictures across all conditions were included in analysis of SCRs for the picture period. In addition, because of a concern that SCRs to IAPS pictures would attenuate over time (thus reducing our ability to detect effects related to valence and uncertainty), we compared the total number of SCRs to pictures in early and late runs. For the 44 participants who met the above $25 \%$ response cut-off across all four runs, the mean number of SCRs dropped from 21.6 in Runs 1 to 2 to 14.0 in Runs 3 to $4, t(43)=7.105, p<$ .001 . Given this $35 \%$ reduction of responses, SCR analyses were conducted on data from Runs 1 to 2 only for the 52 participants with suprathreshold SCRs to at least $25 \%$ of the pictures in those runs (at least 12 responses to a total of 48 pictures). In addition to analysis of SCRs to pictures, we also analyzed SCRs during the cue period for the 41 participants with suprathreshold SCRs to at least $25 \%$ of the cues in Runs 1 to 2 .

Statistical analyses for SCR data were analogous to those described above for online ratings of negative affect. To test the hypothesis that uncertainty would be associated with heightened SCRs after aversive pictures, a paired two-sample $t$ test collapsed across groups compared SCRs with aversive pictures preceded by certain versus uncertain cues. To test whether the act of reporting expectancies would have an impact on these responses, SCR data were also submitted to a Group $(\mathrm{ER}, \mathrm{NER}) \times$ Certainty (Certain, Uncertain) $\times$ Picture Valence (Aversive, Neutral) ANOVA. Anticipatory SCR data were analyzed with a Group (ER, NER) $\times$ Cue Type ("X," "?," "O") ANOVA. Although the anticipatory SCRs in the ER group may have been affected by concurrent manipulation of the dial, we expected to see greater SCRs for the "X" than "O" cue, with intermediate SCRs for the "?" cue, consistent with previous work (Dunsmoor, Bandettini, \& Knight, 2007).

\section{Results}

\section{Expectancy Ratings and Postexperiment Estimates}

Participants in the ER group demonstrated an a priori expectancy bias, in that expectancy ratings following the first "?" cue were greater than $50 \%$, the point on the rating scale labeled "uncertain" $(M=55.0 \%, S D=15.0 \% ; t(38)=2.065, p=.046$, $d=.33$ ). Online expectancy ratings for all subsequent "?"cues in the experiment were greater than $50 \%$, reflecting an online expectancy bias (see Figure 2$)(M=54.1 \%, S D=11.6 \% ; t(38)=$ $2.207, p=.033, d=.35)$. One-sample $t$ tests of online expectancy versus $50 \%$ for each run separately showed a significant expec-

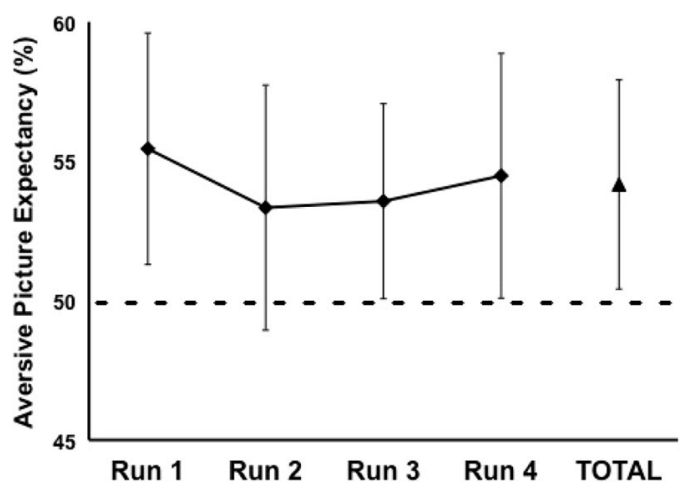

Figure 2. Mean expectancy ratings for the Ratings group $(N=39)$ after the uncertain ("'?") cue for each of four experimental runs. Error bars depict $95 \%$ confidence intervals around the mean. 
tancy bias for Runs 1, 3, and 4 (all $t \mathrm{~s}>2.0$, all $p \mathrm{~s}<.05$, all $d \mathrm{~s}>$ .40 , for Run $2, t(38)=1.520, p=.137, d=.39$ ). There was no significant linear trend of time on expectancy ratings for the "?" cue $\left(F(1,38)=.342, p=.562\right.$, partial $\left.\eta^{2}=.061\right)$.

Postexperiment estimates of the relationship between "?" cues and aversive pictures were not different from the true ratio of $50 \%$ $(M=48.6 \%, S D=12.1 \% ; t(77)=-1.410, p=.307, d=.12)$, and the ER and NER groups did not differ in these estimates, $t(76)=.840, p=.404, d=.17$. There were, however, notable individual differences in these estimates, with a range of $20 \%$ to $80 \%$, and statistically equivalent proportions of participants estimating 50\% (25/78 participants), less than 50\% (30/78), and greater than $50 \%(23 / 78), \chi^{2}(2, N=78)=1.00, p=.6065$. To examine potential factors contributing to this high variability, an exploratory analysis was conducted using simultaneous multiple linear regression to predict these postexperiment estimates. A priori expectancy ratings for the "?" cue and mean online expectancy ratings for the "?" cue were both entered as predictors because these measures have previously shown associations with postexperiment covariation estimates (Amin \& Lovibond, 1998; de Jong et al., 1995, 1998). Total scores for the IUS were also entered because dispositional attitudes toward uncertainty could affect participants' estimates of the relationship between uncertain cues and aversive outcomes. To test whether subjective aversiveness of the IAPS slides might affect these postexperiment estimates, the final predictor was the total number of aversive pictures that were rated by each participant during the experiment as being at all unpleasant (i.e., picture rating $<0.0)^{3}$. All independent variables were mean-centered before being entered into the regression equation.

Online expectancy ratings and the number of pictures rated as unpleasant were significant predictors of postexperiment estimates, whereas a priori expectancy ratings and IU were not (see Tables 1 and 2, Figure 3). Increased online expectancies that aversive pictures would follow the "?" cue were associated with higher postexperiment estimates of the "?" cue being followed by aversive pictures (partial $r=.530, p=.0004$ ) (see Figure 3A). In addition, participants who rated more of the aversive pictures as unpleasant reported higher postexperiment estimates (partial $r=$ $.347, p=.0283)$ (see Figure 3B).

\section{Impact of Uncertainty on Affective Responses to Aversive Pictures}

Skin conductance data. SCRs to aversive pictures preceded by "?" cues were larger than SCRs to aversive pictures preceded

Table 1

Simultaneous Multiple Regression Analysis for Variables

Predicting Postexperiment Estimates of Uncertain Cue/Aversive Picture Relationship $(N=39)$

\begin{tabular}{lrrr}
\hline \multicolumn{1}{c}{ Independent variable } & $B$ & $S E B$ & $\beta$ \\
\hline Intolerance of uncertainty & .000 & .001 & -.051 \\
A priori expectancy & -.159 & .123 & -.204 \\
Online expectancy & .551 & .151 & $.561^{* * *}$ \\
No. pictures rated unpleasant & .007 & .003 & $.307^{*}$ \\
\hline
\end{tabular}

Note. Full model $R^{2}=.323, F(4,34)=4.062, p=.008$

${ }^{*} p<.05$. ${ }^{* * *} p<.005$. by "X" cues (see Figure 4, Table 3) $(t(51)=2.403, p=.020, d=$ .52). The analogous effect of certainty for SCRs to neutral pictures was not significant, $t(51)=1.304, p=.198, d=.42$. To test for between-groups differences in SCRs to pictures, we conducted a repeated-measures Group $\times$ Certainty $\times$ Picture Type ANOVA. ${ }^{4}$ This analysis revealed main effects of Certainty $(F(1,50)=5.96$, $p=.018$, partial $\left.\eta^{2}=.107\right)$ and Picture Type $(F(1,50)=85.24$, $p<.001$, partial $\left.\eta^{2}=.630\right)$. SCRs were greater for pictures preceded by uncertain relative to certain cues and for aversive relative to neutral pictures. The Certainty $\times$ Picture Type interaction was not significant $\left(F(1,50)=0.41, p=.525\right.$, partial $\eta^{2}=$ .008), indicating comparable effects of uncertainty on SCRs to aversive and neutral pictures. There were no significant effects involving Group. None of the three bias measures were correlated with SCRs to pictures (all $p \mathrm{~s}>.40$ ).

For SCRs to anticipatory cues, a repeated-measures Group $\times$ Cue Type ANOVA revealed a main effect of Cue Type $(F(2$, $78)=8.393, p<.001$, partial $\eta^{2}=.177$ ). Consistent with previous findings (Dunsmoor et al., 2007), SCRs to the " $X$ " cue were greater than SCRs to the "?" cue, $t(40)=2.787, p=.008$, $d=.88$ and SCRs to the "O" cue, $t(40)=3.933, p<.001, d=$ 1.50. There was a trend for larger SCRs to the "?" cue relative to the "O" cue, $t(40)=1.769, p=.085, d=.65$, providing some support for the proposal that a cue representing uncertainty about potential aversive events can be fear-relevant.

Mood rating data. Consistent with the findings for SCR data, mood ratings for aversive pictures preceded by the uncertain cue $(M=-1.055, S D=1.687)$ were more negative than mood ratings for aversive pictures preceded by the certain cue $(M=$ $-0.905, S D=1.587), t(77)=2.280, p=.025, d=.20 . \mathrm{A}$ repeated-measures Group $\times$ Certainty $\times$ Picture Type ANOVA identified a main effect of Picture Type on participants' selfreported $\operatorname{mood}\left(F(1,76)=134.601, p<.001\right.$, partial $\left.\eta^{2}=.639\right)$, such that individuals reported their mood as more negative after aversive relative to neutral pictures. A marginally significant Certainty $\times$ Picture Type interaction $(F(1,76)=3.649, p=.060$, partial $\left.\eta^{2}=.046\right)$ indicated that the above effect of uncertainty on mood ratings after aversive pictures did not extend to mood ratings after neutral pictures, $t(77)=-0.276, p=.784$, partial $\eta^{2}=.001$. The only other effect was a Group $\times$ Certainty $\times$ Picture Type interaction $\left(F(1,76)=4.800, p=.032\right.$, partial $\left.\eta^{2}=.059\right)$. Decomposition of this three-way interaction showed that the ER and NER groups had comparable mood ratings for aversive pic-

\footnotetext{
${ }^{3}$ The 0.0 cut-off was selected because a) the middle point of the scale was marked as "neutral," meaning that any ratings to the left of this point can reasonably be interpreted as more unpleasant than neutral, and b) a cut-off less than 0.0 resulted in a restricted range in the number of aversive pictures rated as unpleasant.

${ }^{4}$ It should be noted that this ANOVA is not balanced with regard to the number of events in each "Certainty" cell (2/3 of trials can be classified as "certain," whereas only $1 / 3$ are "uncertain"). Although the DV is the mean SCR across trials, this unbalanced design presumably results in a greater degree of measurement error for "uncertain" trials. This issue arises again when analogous ANOVAs are used to compare mood and picture ratings for pictures after certain versus uncertain cues. The results of all such analyses are presented with this caveat, although the upshot of this design is likely to be only a reduction in power and not a biasing of results in any systematic way.
} 
Table 2

Pearson Product-Moment Correlations for Variables in Simultaneous Multiple Regression Analysis $(N=39)$

\begin{tabular}{lcccc}
\hline \multicolumn{1}{c}{ Variable } & $\begin{array}{c}\text { A priori } \\
\text { expectancy }\end{array}$ & $\begin{array}{c}\text { Online } \\
\text { expectancy }\end{array}$ & $\begin{array}{c}\text { No. pictures } \\
\text { aversive }\end{array}$ & $\begin{array}{c}\text { Postexperiment } \\
\text { estimates }\end{array}$ \\
\hline $\begin{array}{l}\text { Intolerance of Uncertainty } \\
\text { A priori expectancy }\end{array}$ & $.260^{*}$ & .110 & -.030 & -.051 \\
Online expectancy & & $.377^{* *}$ & .016 & -.001 \\
No. pictures rated aversive & & & -.120 & $.441^{* * * *}$ \\
\hline
\end{tabular}

${ }^{*} p<.10 . \quad{ }^{* * *} p<.01 . \quad{ }^{* * *} p<.005$.

tures (allowing the above planned contrast collapsing across groups), but the ER group showed a trend toward more positive mood after neutral pictures preceded by "O" relative to "?" cues, $t(38)=1.771, p=.085, d=.25$, whereas the NER group showed the opposite pattern, $t(38)=-2.040, p=.048, d=.34$.

Picture rating data. For trials on which participants rated the valence of the pictures rather than their mood, there were no effects of cue certainty on valence ratings for aversive pictures, $t(77)=-1.212, p=.229, d=.27$ or neutral pictures, $t(77)=$
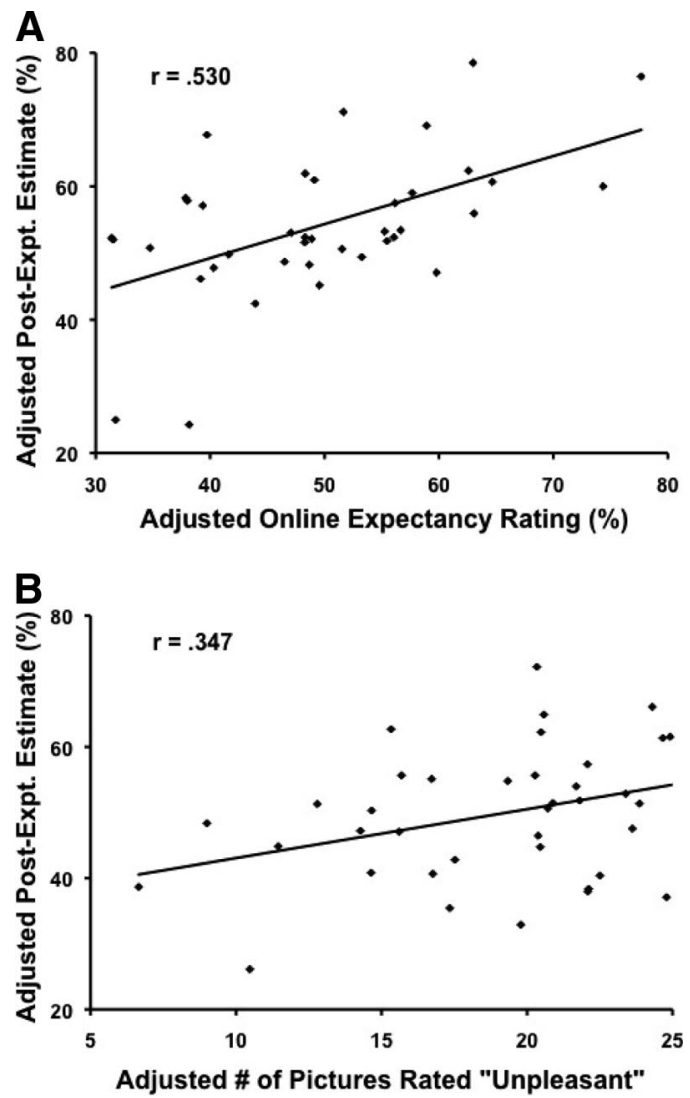

Figure 3. (A) Plot of postexperiment estimates and online expectancy ratings, after partialing out variance attributed to Intolerance of Uncertainty (IU), a priori expectancies, and the number of pictures rated as unpleasant. (B) Plot of postexperiment estimates and the number of aversive pictures rated as unpleasant, after partialing out variance attributed to IU, a priori expectancies, and online expectancies.
$-0.463, p=.644, d=.10$. A repeated-measures Group $\times$ Certainty $\times$ Picture Type ANOVA for these picture ratings revealed only a main effect of Picture Type $(F(1,76)=523.65, p<$ .001 , partial $\left.\eta^{2}=.873\right)$, such that individuals rated aversive pictures as more negative than neutral pictures.

\section{Discussion}

This experiment was designed to investigate biased expectancies for cues indicating uncertainty about the subsequent occurrence of aversive pictures, as well as the impact of uncertainty on autonomic and self-reported responses to those pictures. Corroborating skin conductance and self-report data showed that the negative impact of aversive events was amplified when such events occurred in an uncertain context. Similarly, participants demonstrated biased a priori and online expectancies of aversive pictures after uncertain cues, providing support for the idea that uncertainty can serve as a fear-relevant cue that is likely to be overassociated with aversive events. The magnitude of this online expectancy bias uniquely predicted participants' postexperiment estimates of the relationship between uncertain cues and aversive pictures. Finally, a direct comparison of participants who did and did not provide online expectancy ratings failed to identify significant group differences in self-report or skin conductance data, providing justification for the use of such ratings in fear conditioning and illusory correlation paradigms.

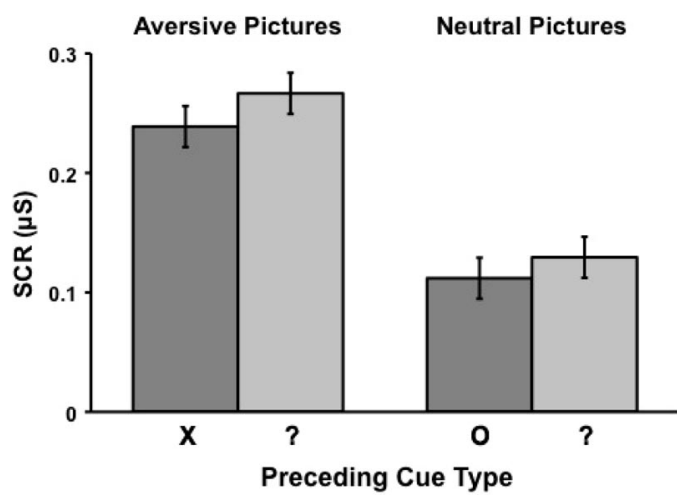

Figure 4. Mean skin conductance responses (SCRs) to aversive and neutral pictures preceded by cues indicating either certainty ("X"/"O") or uncertainty ("'?") about the valence of the following picture. Error bars represent $95 \%$ confidence intervals around the mean after adjusting for between-subjects variance (Loftus \& Masson, 1994). 
Table 3

Mean $( \pm S D)$ Skin Conductance Responses to Pictures $(N=52)$

\begin{tabular}{lccc}
\hline & \multicolumn{3}{c}{ Preceding cue type } \\
\cline { 2 - 4 } Picture valence & Certain & Uncertain & Combined \\
\hline Aversive & $.238 \pm .114$ & $.267 \pm .127$ & $.248 \pm .112^{\mathrm{a}}$ \\
Neutral & $.112 \pm .080$ & $.129 \pm .103$ & $.117 \pm .075^{\mathrm{a}}$ \\
Combined & $.175 \pm .081$ & $.198 \pm .097$ & $.183 \pm .081$ \\
\hline
\end{tabular}

Note. All values are in units of $\mu \mathrm{S}$. Skin conductance responses were square root-transformed before calculation of means.

a Because each picture type occurred twice as frequently after the "Certain" as opposed to the "Uncertain" cue, marginal means for responses to Aversive and Neutral pictures are a weighted average of cell means (i.e., $2 / 3 *$ Certain mean $+1 / 3 *$ Uncertain mean).

A number of parallels exist between this study and fear conditioning research. In a typical fear conditioning paradigm, multiple pairings of a neutral stimulus (CS+) with an aversive outcome (UCS) result in elevated fear responses upon presentation of the $\mathrm{CS}+$ alone. Instructed fear conditioning paradigms make the CS+/UCS relationship explicit to participants at the outset, as was done in the current study. A consistent finding in conditioning studies is unconditioned response (UCR) diminution - that is, a reduction in the magnitude of the fear response to the UCS when the UCS occurs predictably (e.g., a UCS on $100 \%$ vs. $50 \%$ of CS + trials; Dunsmoor et al., 2008; Grings \& Schell, 1971; Kimmel, 1967; Lykken, Macindoe, \& Tellegen, 1972; Peeke \& Grings, 1968). Consistent with this literature, we observed smaller SCRs to aversive pictures occurring in the context of certainty relative to uncertainty. In addition, self-reported mood was more negative for aversive pictures preceded by the uncertain as opposed to the certain cue. These findings are in line with our recent fMRI study using a similar paradigm (Sarinopoulos et al., 2010), in which aversive pictures preceded by certain, relative to uncertain, cues were associated with diminished activity in the insula and amygdala, two regions generally involved in responding to aversive stimuli across modalities (Craig, 2009; Davis \& Whalen, 2001; Dunsmoor et al., 2008; Nitschke et al., 2006a; Nitschke et al., 2006b; Ploghaus et al., 1999; Sarinopoulos et al., 2006). These regions are also consistently implicated in human fear conditioning studies (Critchley, Mathias, \& Dolan, 2002; Sehlmeyer et al., 2009). Of note, the above UCR diminution studies used aversive stimuli of a relatively basic nature, such as aversive shocks or sounds. Our findings for aversive pictures extend the scope of this phenomenon, suggesting that fear responses to more complex and ecologically valid stimuli can also be reduced if individuals are able to better predict their occurrence.

The SCR diminution in the current study may reflect automatic or more effortful regulation of emotional responses to aversive events that participants are certain will appear (Gross, 1998). Alternatively, the uncertain context may elevate participants' anxiety and result in potentiated SCRs to aversive pictures, relative to such pictures that occur predictably and with certainty (Lang, Bradley, \& Cuthbert, 1990). Whether the current results reflect certainty-influenced diminution or uncertainty-related potentiation, the SCR and mood findings reported here are consistent with a number of reports suggesting that uncertainty adversely affects mood and heightens the negative impact of aversive stimuli (Bar-
Anan, Wilson, \& Gilbert, 2009; Hirsh \& Inzlicht, 2008; Kimmel, 1967; Nader \& Balleine, 2007; Schell \& Grings, 1971).

This view of uncertainty as an aversive state was further demonstrated by participants' biased expectancies of aversive pictures after uncertain cues. Aversive picture expectancies were significantly greater than chance at the first presentation of the uncertain cue, reflecting an a priori expectancy bias related to uncertainty. Online expectancy biases were also observed throughout the experiment despite ample opportunity for participants to correct their biased estimates through repeated cue-picture pairings. A number of previous studies have identified both a priori and online biases in expectancies of aversive stimuli after fear-relevant cues (e.g., Amin \& Lovibond, 1997; Davey, 1992; de Jong et al., 1995; Mühlberger et al., 2006). The consistency between the current results and previous results for expectancy biases related to fearrelevant cues provides further support for the notion that a cue explicitly representing uncertainty can serve a role akin to that of fear-relevant stimuli in illusory correlation paradigms. Because aversive events that occur under conditions of uncertainty have a more negative impact than certain aversive events, individuals may learn to evaluate uncertainty more negatively, thus contributing to the overassociation of uncertainty with negative outcomes.

Despite the presence of a priori and online expectancy biases, there was no postexperiment bias in estimates of the relationship between the "?" cue and aversive pictures. These unbiased estimates are at odds with the findings of Sarinopoulos et al. (2010), who demonstrated a significant bias for estimates of this cuepicture relationship (mean estimate $=63.7 \%$ ) using a very similar task and postexperiment questionnaire. One potential explanation for the accurate estimates observed in the current study is that the aversive pictures were not perceived as being particularly unpleasant by the current cohort of individuals, as reported by many of the participants during postexperiment interviews. Indeed, participants' average valence ratings of aversive pictures in the previous study were a full point more unpleasant than in the current study. The covariation bias has been proposed to reflect an "affective match" between fear-relevant cues and subsequent aversive outcomes (Tomarken, Sutton, \& Mineka, 1995; see also Lang et al., 1990), and the absence of strong affective responses to aversive pictures in the current study may have resulted in accurate postexperiment estimates. Of note, the observation of biased online estimates suggests that expectancy ratings are more sensitive to aversive stimuli that are perceived as less unpleasant than are retrospective contingency estimates.

Consistent with this "affective match" hypothesis (Tomarken et al., 1995), the number of aversive pictures rated by each participant as unpleasant uniquely predicted postexperiment estimates, suggesting that the use of a more aversive stimulus might generate stronger affective responses and biased postexperiment estimates in more participants. Postexperiment estimates were also uniquely predicted by participants' online expectancies after uncertain cues, consistent with previous illusory correlation paradigms that have compared online and postexperiment ratings (Amin \& Lovibond, 1997; de Jong \& Merckelbach, 1990; de Jong et al., 1995, 1998). These findings that online expectancies and perceived unpleasantness of the aversive pictures were able to explain the large variability in postexperiment bias highlight the importance of considering individual differences when designing experiments and conducting analyses. Given the broad range of emotional re- 
sponses to aversive events within even nonclinical samples, robust and potentially important effects may emerge only through analyses that highlight individual differences rather than considering variability across participants as noise to be eliminated.

In this first study to directly test the potential impact of online expectancy ratings on subsequent physiological or self-reported responses (cf. Arkes \& Harkness, 1983, Experiment 7), there was a striking lack of differences between groups that did and did not report online expectancy ratings after cue presentations. No group differences were observed for SCRs to pictures or cues, mood or picture ratings after aversive pictures, or postexperiment estimates of the percentage of uncertain cues followed by aversive pictures. Online expectancy ratings are widely used as a measure of participants' awareness of contingencies in studies investigating conditioned learning (Dunsmoor et al., 2007, 2008; Knight, Cheng, Smith, Stein, \& Helmstetter, 2004; Lovibond \& Shanks, 2002) and illusory correlations (de Jong \& Merckelbach, 1990; de Jong et al., 1995, 1998; de Jong \& Peters, 2007; Garner, Mogg, \& Bradley, 2006; Hermann et al., 2004; Pauli, Montoya, \& Martz, 1996). Recent research suggests that subjective contingency awareness, as reflected in online expectancy ratings, is dissociable from fear responding as assayed by measures such as fear-potentiated startle (Kindt, Soeter, \& Vervliet, 2009; Soeter \& Kindt, 2010). Those recent findings highlight the importance of collecting a measure of subjective contingency awareness in addition to physiological measures of fear responding, and our findings here suggest that such measures may be collected without affecting other measures of interest.

Expectancy ratings may provide insight into internal processes without noticeably affecting participants' task performance, behavior, or peripheral physiology. When individuals are faced with uncertainty, a common and seemingly automatic coping strategy is to rate the relative frequencies of subsequent events (Volz, Schubotz, \& von Cramon, 2003). If participants are already estimating the likelihood of an aversive picture when presented with the uncertain cue, asking them to report this estimate would not be expected to have an effect on the task at hand (see Ericsson \& Simon, 1980). Our findings provide justification for the continued use of online self-reports of participants' thoughts or feelings, so long as such reports reflect information that is easily accessible and task-relevant. Such self-report data could be especially beneficial, for example, in helping to curb "reverse inference" problems that plague many neuroimaging studies - that is, inferring a specific psychological state from a pattern of neural activation alone, based on previous research reporting associations between that state and similar activation (Carter \& Pine, 2006; van Horn \& Poldrack, 2009). A final note is that the lack of group differences here, and null findings in general, must always be interpreted with caution because the lack of significant group differences in a given sample may simply reflect a lack of statistical power to identify true differences in the population.

A richer understanding of the relationship between uncertainty and expectancies of negative outcomes may provide novel insight into the pathology of anxiety disorders. A key feature shared across the anxiety disorders is a tendency to show excessive and maladaptive anticipation of negative future events (Barlow, 2002; Nitschke \& Heller, 2005; Nitschke et al., 2009). This anticipation is especially heightened when future events are uncertain or unpredictable, leading to models that stress intolerance of uncertainty as a contributing factor to anxiety (Boelen \& Reijntjes, 2009; Dugas et al., 1998; Holaway, Heimberg, \& Coles, 2006). Illusory correlation paradigms in general have a degree of uncertainty intrinsically involved because the relationships between various cues and aversive/safe outcomes are not conveyed to participants. The current research, however, explicitly probes individuals' expectancies and associations related to uncertainty and juxtaposes anticipation under certain and uncertain conditions to quantify the direct consequences of uncertainty on responses to negative outcomes.

Extensions of this work in anxiety disorders could identify whether patients demonstrate heightened expectancy biases related to uncertainty, or whether biased postexperiment estimates are seen in these individuals. It would be especially informative to explore the consequences of biased estimates of aversion related to uncertainty by relating such biases to symptomology or treatment outcome. Previous research has shown that expectancy biases for combat-related pictures were predictive of posttraumatic stress disorder symptoms after combat exposure (Engelhard et al., 2009), and heightened covariation bias in phobic women after treatment was associated with an increased likelihood of relapse (de Jong et al., 1995). Work probing biases related to the state of uncertainty could similarly identify individuals at risk of developing an anxiety disorder or suffering relapse, and interventions could target negative attitudes toward uncertainty or promote strategies leading to accurate assessment of contingencies. By investigating biases related to uncertainty and identifying how they change over time, researchers can lay the groundwork for improved treatment that is "directed toward the appropriate cognitive target: preexisting beliefs concerning aversive outcomes, versus the tendency to process events online in such a way as to exaggerate threat" (Amin \& Lovibond, 1997, p. 287).

A primary limitation of this study is that although this sample was relatively diverse with regard to race, participants were primarily young university students. This raises questions of whether findings can be generalized to other groups, especially given age-related differences in responding to ambiguous emotional information (van Reekum et al., 2010). An important future direction for this area of research is to include multiple uncertain cues with varying associated probabilities of negative outcomes (Bach et al., 2009; Volz et al., 2003). This manipulation of probability would also allow for a comparison of responses to negative events occurring under conditions of ambiguity versus high or low risk, thus providing greater insight into what aspects of uncertainty result in enhanced aversive responses.

In summary, this study adds to an extensive body of evidence showing that uncertainty amplifies the negative impact of aversive events, as assessed by both physiological and self-report measures. Our use of online expectancy ratings after certain and uncertain cues showed that individuals have a tendency to overassociate uncertainty with aversive outcomes. This bias was already present at the start of the study, continued throughout the experiment, and contributed to individual differences in biased postexperiment estimates of the relationship between uncertain cues and aversive pictures. Enhanced responses to uncertain (relative to certain) aversive events may lead individuals to associate the state of uncertainty itself with aversion and could thus contribute to the generation of uncertainty-related expectancy biases. Given the evidence linking uncertainty to anxiety disorders and the central 
role of anticipatory dysfunction in these individuals, investigations of expectancy biases related to uncertainty may provide novel insight into the cognitive mechanisms underlying anxiety pathology.

\section{References}

Alloy, L. B., \& Tabachnik, N. (1984). Assessment of covariation by humans and animals: The joint influence of prior expectations and current situational information. Psychological Review, 91, 112-149.

Amin, J. M., \& Lovibond, P. F. (1997). Dissociations between covariation bias and expectancy bias for fear-relevant stimuli. Cognition and Emotion, 11, 273-289.

Arkes, H. R., \& Harkness, A. R. (1983). Estimates of contingency between two dichotomous variables. Journal of Experimental Psychology: General, 112, 117-135.

Atlas, L. Y., Bolger, N., Lindquist, M. A., \& Wager, T. D. (2010). Brain mediators of predictive cue effects on perceived pain. Journal of Neuroscience, 30, 12964-12977.

Bach, D. R., Seymour, B., \& Dolan, R. J. (2009). Neural activity associated with the passive prediction of ambiguity and risk for aversive events. Journal of Neuroscience, 29, 1648-1656.

Bar-Anan, Y., Wilson, T. D., \& Gilbert, D. T. (2009). The feeling of uncertainty intensifies affective reactions. Emotion, 9, 123-127.

Barlow, D. H. (2002). Anxiety and its disorders: The nature and treatments of anxiety and panic (2nd ed.). New York: Guilford Press.

Boelen, P. A., \& Reijntjes, A. (2009). Intolerance of uncertainty and social anxiety. Journal of Anxiety Disorders, 23, 130-135.

Borkovec, T. D., \& Inz, J. (1990). The nature of worry in generalized anxiety disorder: A predominance of thought activity. Behaviour Research and Therapy, 28, 153-158.

Buhr, K., \& Dugas, M. J. (2002). The intolerance of uncertainty scale: Psychometric properties of the English version. Behaviour Research and Therapy, 40, 931-945.

Carter, C. S., \& Pine, D. S. (2006). Polishing the windows of the mind. American Journal of Psychiatry, 163, 761-763.

Chapman, L. J., \& Chapman, J. P. (1967). Genesis of popular but erroneous psychodiagnostic observations. Journal of Abnormal Psychology, 72, 193-204.

Chapman, L. J., \& Chapman, J. P. (1969). Illusory correlation as an obstacle to the use of valid psychodiagnostic signs. Journal of Abnormal Psychology, 74, 271-280.

Craig, A. (2009). How do you feel - now? The anterior insula and human awareness. Nature Reviews Neuroscience, 10, 59-70.

Critchley, H. D., Mathias, C. J., \& Dolan, R. J. (2002). Fear conditioning in humans: The influence of awareness and autonomic arousal on functional neuroanatomy. Neuron, 33, 653-663.

Davey, G. C. L. (1992). An expectancy model of laboratory preparedness effects. Journal of Experimental Psychology: General, 121, 24-40.

Davey, G. C. L. (2006). Cognitive mechanisms in fear acquisition and maintenance. In M. G. Craske, D. Hermans, \& D. Vansteenwegen, (Eds.), Fear and learning: From basic processes to clinical implications (pp. 99-116). Washington, DC: American Psychological Association.

Davey, G. C. L., \& Dixon, A. L. (1996). The expectancy bias model of selective associations: The relationship of judgments of CS dangerousness, CS-UCS similarity and prior fear to a priori and a posteriori covariation assessments. Behaviour Research and Therapy, 34, 235252.

Davis, M., \& Whalen, P. (2001). The amygdala: Vigilance and emotion. Molecular Psychiatry, 6, 13-34.

Dawson, M. E., Schell, A. M., \& Filion, D. L. (2007). The electrodermal system. In J. T. Cacioppo, L. G. Tassinary, \& G. G. Berntson, (Eds.), Handbook of Psychophysiology (3rd ed., pp. 159-181). New York: Cambridge University Press. de Araujo, I. E., Rolls, E. T., Velazco, M. I., Margot, C., \& Cayeux, I. (2005). Cognitive modulation of olfactory processing. Neuron, 46, 671679.

de Jong, P. J., \& Merckelbach, H. (1990). Illusory correlation, on-line probability estimates, and electrodermal responding in a (quasi)conditioning paradigm. Biological Psychology, 31, 201-212.

de Jong, P. J., Merckelbach, H., \& Arntz, A. (1995). Covariation bias in phobic women: The relationship between a priori expectancy, on-line expectancy, autonomic responding, and a posteriori contingency judgment. Journal of Abnormal Psychology, 104(1), 55-62.

de Jong, P. J., Merckelbach, H., Bögels, S., \& Kindt, M. (1998). Illusory correlation and social anxiety. Behaviour Research and Therapy, 36, 1063-1073.

de Jong, P. J., \& Peters, M. L. (2007). Blood-injection-injury fears: Harmvs. disgust-relevant selective outcome associations. Journal of Behavior Therapy and Experimental Psychiatry, 38, 263-274.

de Jong, P. J., van den Hout, M. A., \& Merckelbach, H. (1995). Covariation bias and the return of fear. Behaviour Research and Therapy, 33(2), 211-213.

Dugas, M. J., Gagnon, F., Ladouceur, R., \& Freeston, M. H. (1998). Generalized anxiety disorder: A preliminary test of a conceptual model. Behaviour Research and Therapy, 36, 215-226.

Dunsmoor, J. E., Bandettini, P. A., \& Knight, D. C. (2007). Impact of continuous versus intermittent CS-UCS pairing on human brain activation during Pavlovian fear conditioning. Behavioral Neuroscience, 121, 635-642.

Dunsmoor, J. E., Bandettini, P. A., \& Knight, D. C. (2008). Neural correlates of unconditioned response diminution during Pavlovian conditioning. Neurolmage, 40, 811-817.

Engelhard, I. M., de Jong, P. J., van den Hout, M. A., \& van Overveld, M. (2009). Expectancy bias and the persistence of posttraumatic stress. Behaviour Research and Therapy, 47, 887-892.

Ericsson, K. A., \& Simon, H. A. (1980). Verbal Reports as Data. Psychological Review, 87(3), 215-251.

Etkin, A., Egner, T., Peraza, D., Kandel, E., \& Hirsch, J. (2006). Resolving emotional conflict: A role for the rostral anterior cingulate cortex in modulating activity in the amygdala. Neuron, 51, 871-882.

Etkin, A., Prater, K. A., Hoeft, F., Menon, V., \& Schatzberg, A. F. (2010). Failure of anterior cingulate activation and connectivity with the amygdala during implicit regulation of emotional processing in generalized anxiety disorder. American Journal of Psychiatry, 167, 545-554.

Etkin, A., \& Wager, T. (2007). Functional neuroimaging of anxiety: A meta-analysis of emotional processing in PTSD, social anxiety disorder, and specific phobia. American Journal of Psychiatry, 164, 1476-1488.

Garner, M., Mogg, K., \& Bradley, B. P. (2006). Fear-relevant selective associations and social anxiety: Absence of a positive bias. Behaviour Research and Therapy, 44, 201-217.

Grings, W. W., \& Schell, A. M. (1971). Effects of trace versus delay conditioning, interstimulus interval variability, and instructions of UCR diminution. Journal of Experimental Psychology, 90, 136-140.

Gross, J. J. (1998). The emerging field of emotion regulation: An integrative review. Review of General Psychology, 2, 271-299.

Hermann, C., Ofer, J., \& Flow, H. (2004). Covariation bias for ambiguous social stimuli in generalized social phobia. Journal of Abnormal Psychology, 113, 646-653.

Hirsh, J. B., \& Inzlicht, M. (2008). The devil you know: Neuroticism predicts neural response to uncertainty. Psychological Science, 19, 962 967.

Holaway, R. M., Heimberg, R. G., \& Coles, M. E. (2006). A comparison of intolerance of uncertainty in analogue obsessive-compulsive disorder and generalized anxiety disorder. Journal of Anxiety Disorders, 20, $158-174$.

Hsu, M., Bhatt, M., Adolphs, R., Tranel, D., \& Camerer, C. F. (2005). 
Neural systems responding to degrees of uncertainty in human decisionmaking. Science, 310, 1680-1683.

Kagan, J. (1972). Motives and development. Journal of Personality and Social Psychology, 22, 51-66.

Kennedy, S. J., Rapee, R. M., \& Mazurski, E. J. (1997). Covariation bias for phylogenetic versus ontogenetic fear-relevant stimuli. Behaviour Research and Therapy, 35, 415-422.

Kimmel, E. (1967). Judgments of UCS intensity and diminution of UCR in classical GSR conditioning. Journal of Experimental Psychology, 73, 532-543.

Kindt, M., Soeter, M., \& Vervliet, B. (2009). Beyond extinction: Erasing human fear responses and preventing the return of fear. Nature Neuroscience, 12, 256-258.

Kirk, U., Skov, M., Hulme, O., Christensen, M. S., \& Zeki, S. (2009). Modulation of aesthetic value by semantic context: An fMRI study. NeuroImage, 44, 1125-1132.

Knight, D. C., Cheng, D. T., Smith, C. N., Stein, E. A., \& Helmstetter, F. J. (2004). Neural substrates mediating human delay and trace fear conditioning. Journal of Neuroscience, 24, 218-228.

Lang, P. J., Bradley, M. M., \& Cuthbert, B. N. (1990). Emotion, attention, and the startle reflex. Psychological Review, 97, 377-395.

Lang, P. J., Bradley, M. M., \& Cuthbert, B. N. (2008). International affective picture system (IAPS): Affective ratings of pictures and instruction manual. Tech. Rep. No. A-8. Gainesville, FL: University of Florida.

Loftus, G. R., \& Masson, M. E. J. (1994). Using confidence intervals in within-subject designs. Psychonomic Bulletin \& Review, 1, 476-490.

Lovibond, P. F., \& Shanks, D. R. (2002). The role of awareness in Pavlovian conditioning: Empirical evidence and theoretical implications. Journal of Experimental Psychology: Animal Behavior Processes, 28, 3-26.

Lykken, D. T., Macindoe, I., \& Tellegen, A. (1972). Preception: Autonomic response to shock as a function of predictability in time and locus. Psychophysiology, 9, 318-333.

McNally, R. J., \& Heatherton, T. F. (1993). Are covariation biases attributable to a priori expectancy biases? Behaviour Research and Therapy, $31,653-658$.

Mühlberger, A., Wiedemann, G., Herrman, M. J., \& Pauli, P. (2006). Phylo- and ontogenetic fears and the expectation of danger: Differences between spider- and flight-phobic subjects in cognitive and physiological responses to disorder-specific stimuli. Journal of Abnormal Psychology, 115, 580-589.

Nader, K., \& Balleine, B. (2007). Ambiguity and anxiety: When a glass half full is empty. Nature Neuroscience, 10, 807-808.

Nitschke, J. B., Dixon, G. E., Sarinopoulos, I., Short, S. J., Cohen, J. D., Smith, E. E., . . Davidson, R. J. (2006a). Altering expectancy dampens neural response to aversive taste in primary taste cortex. Nature Neuroscience, 9, 435-442.

Nitschke, J. B., \& Heller, W. (2005). Distinguishing neural substrates of heterogeneity among anxiety disorders. International Review of Neurobiology, 67, 1-42.

Nitschke, J. B., Sarinopoulos, I., Mackiewicz, K. L., Schaefer, H. S., \& Davidson, R. J. (2006b). Functional neuroanatomy of aversion and its anticipation. Neurolmage, 29, 106-116.

Nitschke, J. B., Sarinopoulos, I., Oathes, D. J., Johnstone, T., Whalen, P. J., Davidson, R. J., \& Kalin, N. H. (2009). Anticipatory activation in the amygdala and anterior cingulate in generalized anxiety disorder and prediction of treatment response. American Journal of Psychiatry, 166, 302-310.

Oldfield, R. C. (1971). The assessment and analysis of handedness: The Edinburgh inventory. Neuropsychologia, 9, 97-113.

Pauli, P., Montoya, P., \& Martz, G. E. (1996). Covariation bias in panicprone individuals. Journal of Abnormal Psychology, 105, 658-662.

Pauli, P., Wiedemann, G., Dengler, W., \& Kühlkamp, V. (2001). A priori expectancy bias and its relation to shock experience and anxiety: A naturalistic study in patients with an automatic implantable cardioverter defibrillator. Journal of Behavior Therapy and Experimental Psychiatry, 32, 159-171.

Pauli, P., Wiedemann, G., \& Montoya, P. (1998). Covariation bias in flight phobics. Journal of Anxiety Disorders, 12, 555-565.

Paulus, M. P., \& Stein, M. B. (2006). An insular view of anxiety. Biological Psychiatry, 60, 383-387.

Peeke, S. S., \& Grings, W. W. (1968). Magnitude of UCR as a function of variability in the CS-UCS relationship. Journal of Experimental Psychology, 77, 64-69.

Plassman, H., O’Doherty, J., Shiv, B., \& Rangel, A. (2008). Marketing actions can modulate neural representations of experienced pleasantness. Proceedings of the National Academy of Sciences, U S A, 105, 10501054.

Ploghaus, A., Becerra, L., Borras, C., \& Borsook, D. (2003). Neural circuitry underlying pain modulation: Expectation, hypnosis, placebo. TRENDS in Cognitive Sciences, 7, 197-200.

Ploghaus, A., Tracey, I., Gati, J. S., Clare, S., Menon, R. S., Matthews, P. M., \& Rawlins, J. N. (1999). Dissociating pain from its anticipation in the human brain. Science, 284, 1979-1981.

Sanfey, A. G. (2009). Expectations and social decision-making: Biasing effects of prior knowledge on Ultimatum responses. Mind \& Society, 8, 93-107.

Sarinopoulos, I., Dixon, G. E., Short, S. J., Davidson, R. J., \& Nitschke, J. B. (2006). Brain mechanisms of expectation associated with insula and amygdala response to aversive taste: Implications for placebo. Brain, Behavior and Immunity, 20, 20-32.

Sarinopoulos, I., Grupe, D. W., Mackiewicz, K. L., Herrington, J. D., Lor, M. Y., Steege, E. E., \& Nitschke, J. B. (2010). Uncertainty during anticipation modulates neural responses to aversion in human insula and amygdala. Cerebral Cortex, 20, 929-940.

Schell, A. M., \& Grings, W. W. (1971). Judgment of UCS intensity and diminution of unconditioned GSR. Psychophysiology, 8, 427-432.

Sehlmeyer, C., Schöning, S., Zwitserlood, P., Pfleiderer, B., Kircher, T., Arolt, V., \& Konrad, C. (2009). Human fear conditioning and extinction in neuroimaging: A systematic review. PLoS One, 4, e5865.

Soeter, M., \& Kindt, M. (2010). Dissociating response systems: Erasing fear from memory. Neurobiology of Learning and Memory, 94, 30-41.

Tomarken, A. J., Mineka, S., \& Cook, M. (1989). Fear-relevant selective associations and covariation bias. Journal of Abnormal Psychology, 98, 381-394.

Tomarken, A. J., Sutton, S. K., \& Mineka, S. (1995). Fear-relevant illusory correlations: What types of associations promote judgmental bias? Journal of Abnormal Psychology, 104, 312-326.

Urry, H. L., van Reekum, C. M., Johnstone, T., \& Davidson, R. J. (2009). Individual differences in some (but not all) medial prefrontal regions reflect cognitive demand while regulating unpleasant emotion. NeuroImage, 47, 852-863.

van Horn, J. D., \& Poldrack, R. A. (2009). Functional MRI at the crossroads. International Journal of Psychophysiology, 73, 3-9.

van Reekum, C. M., Schaefer, S. M., Lapate, R. C., Norris, C. J., Greischar, L. L., \& Davidson, R. J. (2010). Aging is associated with positive responding to neutral information but reduced recovery from negative information. Social Cognitive and Affective Neuroscience. Advance online publication. doi: 10.1093/scan/nsq031

Volkow, N. D., Wang, G. J., Ma, Y. M., Fowler, J. S., Wong, C., Jayne, M., ... Swanson, J. M. (2006). Effects of expectation on the brain metabolic responses to methylphenidate and to its placebo in nondrug abusing subjects. NeuroImage, 32, 1782-1792.

Volz, K. G., Schubotz, R. I., \& von Cramon, D. Y. (2003). Predicting events of varying probability: Uncertainty investigated by fMRI. $\mathrm{Neu}$ rolmage, 19, 271-280.

Wager, T. D., \& Nitschke, J. B. (2005). Placebo effects in the brain 
Linking mental and physiological processes. Brain, Behavior and Immunity, 19, 281-282.

Wager, T. D., Rilling, J. K., Smith, E. E., Sokolik, A., Casey, K. L., Davidson, R. J., ... Cohen, J. D. (2004). Placebo-induced changes in fMRI in the anticipation and experience of pain. Science, 303, 11621167.

Wiedemann, G., Pauli, P., \& Dengler, W. (2001). A priori expectancy bias in patients with panic disorder. Journal of Anxiety Disorders, 15, 401412.

Wiens, S., Katkin, E. S., \& Öhman, A. (2003). Effects of trial order and differential conditioning on acquisition of differential shock expectancy and skin conductance conditioning to masked stimuli. Psychophysiology, 40, 989-997.

Zubieta, J.-K., Bueller, J. A., Jackson, L. R., Scott, D. J., Xu, Y., Koeppe, R. A., ... Stohler, C. S. (2005). Placebo effects mediated by endogenous opioid activity on $\mu$-opioid receptors. Journal of Neuroscience, 25, $7754-7762$.

Received November 6, 2009

Revision received October 26, 2010

Accepted November 1, 2010 